\title{
Sitzung vom 9. November 1885 .
}

Vorsitzender: Hr. H. Landolt, Vice-Präsident.

Das Protocoll der letzten Sitzung wird genehmigt.

$\mathrm{Zu}$ ausserordentlichen Mitgliedern werden proclamirt die Herren:

G. Benz,
Wilh. Schad, Stuttgart;

Adalbert Linzer, Budapest;

J. Robert Moechel, Philadelphia, Pa.;

J. Block, Göttingen;

Dr. O. Weyl, Mannheim;

Emil Pollak, Neustadt a. d. Hardt;

Aemilius Wunderlich, Würzburg.

$\mathrm{Zu}$ ausserordentlichen Mitgliedern werden vorgeschlagen die Herren :

Jn. Ratnaselacharia, B. A., Principal Mahboot College, Secunderabad, Deccan [India] (durch Ferd. Tiemann und J. Biedermann);

Julius Wertheimer, Esq., B. A. (Lond). Oxford Terrace, St. Helens [Lancashire] (durch Ferd. Tiemann und J. Biedermann);

Dr. John J. Wing, Cambridge, Mass., U. S. A., 39 Brottle St. (durch H. B. Hill und C. L. Jackson); Alexander S. Astufieff, Teikowo [Gouv. Wladimir] (durch

S. Lipkowski und L. Margulies);

Franz Muhlert, Rothestr. 2,
W. Bradley, Obere Rarspüle 16D, $\left\{\begin{array}{c}\text { Göttingen (durch V. } \\ \text { Meyeru.L.Gatter- } \\ \text { mann); }\end{array}\right.$ Helen C. D. Abbott, Philadelphia, Pa. 1509 Lorust St. (durch Ferd. Tiemann und S. P. Sadtler); 
Paul Engländer,

Paul Juillard,

Gustaf Bernhardt,

Eugen Kling,

Genf, Ecole de Chimie

(durch F. Pfaff und S. Levy);

Louis Duparc,

Prof. James M. Crafts, Paris, 175 arenue de Trocadero

(durch W. Gibbs und C. L. Jackson);

K. H. M. van der Zande, Mare 78, Leiden (durch C.

Hensgen und P. van Romburgh;

Friedrich Lau,

Wilh. Deike,

Rostock, Univers.-Labor. (durch

Friedr. Kobbe,

Bernhard Schultz,

O. Jacobsen und R. Hof fmann);

cand. chem. M a r t in I rm i s ch, Annenstr. 20, Berlin $S$.

(durch O. Liebreich und B. Fischer);

Köhler, Assistent bei Dr. C. Bischoff, Berlin (durch C.

Virchow und C. Bischoff);

cand. chem. Friedrich Mayer, Char-

lottenburg, Schlüterstrasse 1a,

cand. chem. W e n s e, Charlottenburg,

Wallstrasse 13,

cand. chem. Otto Salomon, Berlin,

Potsdamerstrasse 27a,

cand. chem. Eugen Alfred Oppelt,

Charlottenburg, Berlinerstrasse 87d,

(durch

C. Liebermann und

S. Kleemann).

Für die Bibliothek sind als Geschenke eingegangen:

144. Annali di chimica medico-farmacentica e di farmacologia. 1885. No. 3. Milano.

1816. Bertoni, Giacomo. Contributo allo studio dell' etereficazione per doppia decomposizione. - Fatti sull' eterificazione per doppia decomposizione.

Der Vorsitzende:

H. L a ndo l t.
Der Schriftführer:

A. Pinner. 